\title{
ENERGY DECAY ESTIMATES FOR A WAVE EQUATION WITH NONLINEAR BOUNDARY FEEDBACK
}

\author{
Mohammad Cherkaoui and Naji Yebari \\ University Moulay Ismail and University Abdelmalek Essadi, Morocco
}

\begin{abstract}
We study a wave equation in one dimensional space with nonlinear dissipative boundary feedback at both ends. We prove existence and uniqueness of solution, strong and uniform exponential decay of energy under some conditions in the nonlinear feedback. Decay rate estimates of the energy are given under weak growth assumptions on the feedback functions.
\end{abstract}

\section{INTRODUCTION AND MAIN RESULTS}

We consider the wave equation with a variable coefficient, controlled at the boundary by the two feedbacks laws $L_{1}$ and $L_{2}$ to be determined in the sequel:

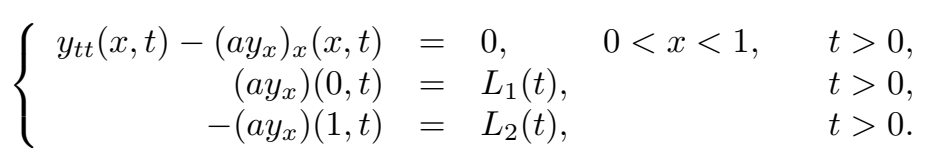

We assume that the function $a($.$) belongs to H^{1}(0,1)$ and that

$$
0<\underline{a} \leq a(x) \text {, for all } x \in[0,1] .
$$

The aim of this paper is to show that the system (1.1) is well posed in the terms of the semigroups of contractions and is asymptotically stabilized by the nonlinear feedback laws $L_{1}$ and $L_{2}$ given as follows:

$$
\begin{aligned}
& L_{1}(t)=k_{p, 0} y(0, t)+k_{v, 0} f\left(y_{t}(0, t)\right), \\
& L_{2}(t)=k_{p, 1} y(1, t)+k_{v, 1} g\left(y_{t}(1, t)\right),
\end{aligned}
$$

2000 Mathematics Subject Classification. 35L05, 35L20, 93D15, 34D20.

Key words and phrases. Wave equation, nonlinear boundary value problems, stabilization of systems by feedback, Lyapunov stability, polynomial decay. 
where $k_{p, 0}, k_{p, 1}, k_{v, 0}$ and $k_{v, 1}$ are non negative constants such that

$$
\begin{aligned}
& k_{v, 0}+k_{v, 1}>0, \\
& k_{p, 0}+k_{p, 1}>0,
\end{aligned}
$$

$f$ and $g$ are suitable nonlinear functions in $C^{0}(\mathbb{R})$.

The boundary stabilization of (1.1) has been studied by many authors. Conrad and Rao [4] have proved that the feedback law

$$
\begin{aligned}
& L_{1}(t)=k_{p, 0} y(0, t)+f\left(y_{t}(0, t)\right), \\
& L_{2}(t)=k_{p, 0} y(1, t)+f\left(y_{t}(1, t)\right), \quad k_{p, 0}>0
\end{aligned}
$$

stabilizes asymptotically the system under a suitable growth condition of $f$. Indeed, in the more general cases for which $f$ is a maximal monotone graph, the strong asymptotic stabilization has been proved by Chen and Wang [2] and Lasieska $[8,9]$.

Let us mention the work of B. Chentouf et al. [1], where a damping model is considered and the equation tolerates a term $\beta y, \beta=$ cte $>0$, the system is asymptotically stabilized by the nonlinear feedback law depending only on the boundary velocities:

$$
\begin{aligned}
L_{1}(t) & =k_{v, 0} f\left(y_{t}(0, t)\right), \\
L_{2}(t) & =k_{v, 1} f\left(y_{t}(1, t)\right), \\
k_{v, 0}+k_{v, 1} & >0
\end{aligned}
$$

under a suitable choice of $f$.

In the linear version of this paper Cherkaoui [3] have proved the strong and uniform exponential decay of the energy and also existence of a Riesz basis associated with a spectral formulation of the problem.

With the feedback laws $L_{1}$ and $L_{2}$ in (1.3), we introduce the energy associated with the system (1.1) as follows:

$$
E(t)=\frac{1}{2} \int_{0}^{1}\left[y_{t}^{2}(x, t)+a(x) y_{x}^{2}(x, t)\right] d x+\frac{1}{2} k_{p, 0} y^{2}(0, t)+\frac{1}{2} k_{p, 1} y^{2}(1, t) .
$$

We derive $E(t)$ with respect to $t$ and integrate by parts, and we show formally that:

$$
E^{\prime}(t)=-k_{v, 0} y_{t}(0, t) f\left(y_{t}(0, t)\right)-k_{v, 1} y_{t}(1, t) g\left(y_{t}(1, t)\right) .
$$

Throughout this paper, both $f$ and $g$ are nondecreasing functions in $C^{0}(\mathbb{R})$ such that

$$
f(0)=g(0)=0, \quad f(s) . s \text { and } g(s) . s>0 \quad \forall s \neq 0 .
$$

Assumption (1.8) implies that the energy $E(t)$ is non-increasing and a Lyapounov function. 
Let us define the Hilbert space $\mathcal{H}=H^{1}(0,1) \times L^{2}(0,1)$ equipped with the inner product:

$(1.9)<(u, v),(w, z)>_{\mathcal{H}}=\int_{0}^{1}\left(a u_{x} w_{x}+v z\right) d x+k_{p, 1} u(1) w(1)+k_{p, 0} u(0) w(0)$.

We consider the following nonlinear operator:

(1.10)

$\mathcal{D}(A)=\left\{(u, v) \in H^{2}(0,1) \times H^{1}(0,1): \begin{array}{r}\left(a u_{x}\right)(0)=k_{p, 0} u(0)+k_{v, 0} f(v(0)) \\ -\left(a u_{x}\right)(1)=k_{p, 1} u(1)+k_{v, 1} g(v(1))\end{array}\right\}$,

and for all $(u, v) \in \mathcal{D}(A)$

$$
A(u, v)=\left(v,\left(a u_{x}\right)_{x}\right)
$$

with the initial data $W_{0}=\left(y_{0}, y_{1}\right)$, the closed-loop system (1.1) can be written as an evolution equation on $\mathcal{H}$ called problem $(\mathrm{P})$

$$
\left\{\begin{aligned}
\dot{W}(t) & =A W(t) \\
W(0) & =W_{0}
\end{aligned}\right.
$$

where $W(t)=\left(y(., t), y_{t}(., t)\right)$. (1.5).

Throughout this paper, $k_{p, 0}$ and $k_{p, 1}$ are assumed to satisfy the hypothesis

Our main results are stated below.

Theorem 1.1. The operator $A: \mathcal{D}(A) \subset \mathcal{H} \longrightarrow \mathcal{H}$ defined by (1.10) and (1.11) generates a $C_{0}$-semigroup of contractions $S(t)$ on the energy space $\mathcal{H}$.

If $k_{v, 0}+k_{v, 1}>0$, then for all initial data $\left(y_{0}, y_{1}\right) \in \mathcal{H}$, the energy $E(t)$ of the problem $(P)$ converges to zero as $t \rightarrow+\infty$.

Theorem 1.2. Assume that $a \in H^{1}(0,1)$ satisfies (1.2) and the hypothesis (1.8) holds.

If $k_{v, 0}+k_{v, 1}>0$ then we have:

(i) If there exist positive constants $C_{1}, C_{2}, C_{3}$ and $C_{4}$ such that for all $x \in \mathbb{R}$

$$
\begin{aligned}
& C_{1}|x| \leq|f(x)| \leq C_{2}|x|, \\
& C_{3}|x| \leq|g(x)| \leq C_{4}|x|,
\end{aligned}
$$

then given any $M>1$, there exists a constant $\omega>0$ such that

$$
E(t) \leq M E(0) e^{-\omega t}, \quad \forall t \geq 0 .
$$

(ii) If there exist positive constants $C_{1}, C_{2}, C_{3}$ and $C_{4}$ and $(p, q) \in\left[1,+\infty\left[^{2}\right.\right.$ with $\max (p, q)=p \vee q>1$ such that for all $x \in \mathbb{R}$,

$$
\begin{aligned}
& C_{1} \min \left(|x|,|x|^{p}\right) \leq|f(x)| \leq C_{2}|x|, \\
& C_{3} \min \left(|x|,|x|^{q}\right) \leq|g(x)| \leq C_{4}|x|,
\end{aligned}
$$


then given any $M>1$, there exists a constant $\omega>0$ depending on $E(0)$ such that

$$
E(t) \leq M E(0)(1+\omega t)^{-\frac{2}{(p \vee q)-1}}, \quad \forall t \geq 0 .
$$

The next section is devoted to the proof of our main results.

\section{Proofs of the MAIN RESUlts}

\subsection{Proof of Theorem 1.1.}

2.1.1. Proof of the well-posedness. By the Lumer-Phillips theorem, it is sufficient to prove that $A$ is an m-dissipative operator.

First, for $w=(y, z) \in D(A)$, we have $A w=\left(z,\left(a y_{x}\right)_{x}\right)$,

$$
\begin{aligned}
\langle A w, w\rangle_{\mathcal{H}} & =\left\langle\left(z,\left(a y_{x}\right)_{x}\right),(y, z)\right\rangle_{\mathcal{H}} \\
& =\int_{0}^{1}\left(a z_{x} y_{x}+\left(a y_{x}\right)_{x} z\right) d x+k_{p, 1} z(1) y(1)+k_{p, 0} z(0) y(0) .
\end{aligned}
$$

Using (1.10), we deduce that

$$
\langle A w, w\rangle_{\mathcal{H}}=-k_{v, 0} z(0) f(z(0))-k_{v, 1} z(1) g(z(1)),
$$

which implies that $A$ is dissipative.

Next, we show the maximality of $A$, i. e. for any given $\left(f_{1}, f_{2}\right) \in \mathcal{H}$, there exists $(u, v) \in \mathcal{D}(A)$ such that $(I-A)(u, v)=\left(f_{1}, f_{2}\right)$. Equivalently, we seek $u$ and $v$ satisfying

$$
\left\{\begin{aligned}
u-v & =f_{1}, \\
v-\left(a u_{x}\right)_{x} & =f_{2}, \\
\left(a u_{x}\right)(0) & =k_{p, 0} u(0)+k_{v, 0} f(v(0)), \\
-\left(a u_{x}\right)(1) & =k_{p, 1} u(1)+k_{v, 1} g(v(1)), \\
u & \in H^{2}(0,1), v \in H^{1}(0,1) .
\end{aligned}\right.
$$

Eliminating the unknown $v$ in equation (2.1), we obtain the following reduced problem

$$
\left\{\begin{aligned}
u-\left(a u_{x}\right)_{x} & =f_{1}+f_{2}=F \in L^{2}(0,1) \\
\left(a u_{x}\right)(0) & =k_{p, 0} u(0)+k_{v, 0} f\left(u(0)-f_{1}(0)\right), \\
-\left(a u_{x}\right)(1) & =k_{p, 1} u(1)+k_{v, 1} g\left(u(1)-f_{1}(1)\right), \\
u & \in H^{2}(0,1) .
\end{aligned}\right.
$$

Now, let us define two functions $F_{0}$ and $F_{1}$ by

$$
F_{0}(x)=k_{v, 0} \int_{0}^{x} f(s) d s \quad \text { and } \quad F_{1}(x)=k_{v, 1} \int_{0}^{x} g(s) d s, \quad \forall x \in \mathbb{R} .
$$

From the hypothesis (1.8), we deduce that $F_{0}$ and $F_{1}$ are two convex functions such that

$$
F_{i} \in C^{1}(\mathbb{R}) ; \quad F_{i}(s) \geq 0, \quad \forall s \in \mathbb{R}, \quad i=0,1 .
$$


In turn, let us define the function $J($.$) on H^{1}(0,1)$ by:

$$
\begin{aligned}
J(w)= & \frac{1}{2} \int_{0}^{1}\left(a w_{x}^{2}+w^{2}\right) d x-\int_{0}^{1} F w d x+\frac{k_{p, 0}}{2} w^{2}(0)+\frac{k_{p, 1}}{2} w^{2}(1) \\
& +F_{0}\left(w(0)-f_{1}(0)\right)+F_{1}\left(w(1)-f_{1}(1)\right) .
\end{aligned}
$$

From (2.4), we deduce that the functional $J($.) is convex, coercive and strongly continuous in the space $H^{1}(0,1)$. Hence there exists a unique function $u \in$ $H^{1}(0,1)$ such that

$$
J(u)=\inf _{w \in H^{1}(0,1)} J(w) .
$$

This implies that the function $\lambda \mapsto J(u+\lambda w)$ admits a minimum at $\lambda=0$ and thus

$$
\left.\frac{d}{d \lambda}[J(u+\lambda w)]\right|_{\lambda=0}=0 \quad \forall w \in H^{1}(0,1),
$$

this means that for any $w \in H^{1}(0,1)$, we have

$$
\begin{aligned}
& \int_{0}^{1} u w d x+\int_{0}^{1} a u_{x} w_{x} d x-\int_{0}^{1} F w d x+k_{p, 0} w(0) u(0)+k_{p, 1} w(1) u(1) \\
& +k_{v, 0} w(0) f\left(u(0)-f_{1}(0)\right)+k_{v, 1} w(1) g\left(u(1)-f_{1}(1)\right)=0
\end{aligned}
$$

In particular for any $w \in C_{0}^{\infty}(0,1)$,

$$
-\int_{0}^{1} a u_{x} w_{x} d x=\int_{0}^{1}[u-F] w d x
$$

this implies the Euler-Lagrange equations

$$
u-\left(a u_{x}\right)_{x}=F \in L^{2}(0,1) .
$$

Then the $H^{2}(0,1)$ regularity follows. Integrating equation (2.6) by parts and using equation (2.7), one obtains

$$
\begin{aligned}
\left(a u_{x}\right)(0) & =k_{p, 0} u(0)+k_{v, 0} f\left(u(0)-f_{1}(0)\right), \\
-\left(a u_{x}\right)(1) & =k_{p, 1} u(1)+k_{v, 1} g\left(u(1)-f_{1}(1)\right) .
\end{aligned}
$$

Therefore, $u$ is the unique solution of system (2.2). Now, we define an element $(u, v)$ by $u$, solution of $(2.2), v=u-f_{1}$, which satisfies clearly system (2.1) and thus $A$ is an m-dissipative operator on $\mathcal{H}$.

Remark 2.1. (i) Given $\left(y_{0}, y_{1}\right) \in \mathcal{D}(A)$, we define

$$
w(t)=\mathcal{S}(t)\left(y_{0}, y_{1}\right)=\left(y(., t), y_{t}(., t)\right) .
$$

Using the regularity result of Haraux [6], we obtain the following smoothness results:

$$
y \in C\left(\mathbb{R}^{+} ; H^{2}(0,1)\right) \cap C^{1}\left(\mathbb{R}^{+} ; H^{1}(0,1)\right) \cap C^{2}\left(\mathbb{R}^{+} ; L^{2}(0,1)\right) .
$$

(ii) If $\left(y_{0}, y_{1}\right) \in \mathcal{H}$, then the Problem $(\mathrm{P})$ admits a unique weak solution

$$
\left(y, y_{t}\right)=\mathcal{S}(t)\left(y_{0}, y_{1}\right) \in C\left(\mathbb{R}^{+} ; \mathcal{H}\right)
$$


and by using again a result of Haraux [6], one obtains

$$
y \in C\left(\mathbb{R}^{+} ; H^{1}(0,1)\right) \cap C^{1}\left(\mathbb{R}^{+} ; L^{2}(0,1)\right) .
$$

2.1.2. Proof of the asymptotic stability. We can assume without loss of generality, that $k_{v, 0}>0$ and $k_{v, 1} \geq 0$. According to the density of $\mathcal{D}(A)$ in $\mathcal{H}$ and the contraction of the semigroup $\mathcal{S}(t)$, it is enough to prove Theorem 1.1 for any initial data $\left(y_{0}, y_{1}\right) \in \mathcal{D}(A)$. Let $\left(y_{0}, y_{1}\right) \in \mathcal{D}(A)$, it is clear that $E(t) \geq 0$ for all $t \geq 0$, and if we set $w=\left(y, y_{t}\right)$ we get

$$
\begin{aligned}
\frac{d E(t)}{d t} & =\left\langle w, \frac{d w}{d t}\right\rangle_{\mathcal{H}}=\langle w, A w\rangle_{\mathcal{H}} \\
& =-k_{v, 0} y_{t}(0, t) f\left(y_{t}(0, t)\right)-k_{v, 1} y_{t}(1, t) g\left(y_{t}(1, t)\right) \leq 0,
\end{aligned}
$$

so, $E(t)$ is a Lyapounov function.

The resolvent of $A$ is compact, and according to Dafermos [5], it follows that the trajectory $O^{+}\left(y_{0}, y_{1}\right)=\left\{\left(y(t), y_{t}(t)\right), t \geq 0.\right\}$ is relatively compact in $E$ for initial data in $\mathcal{D}(A)$. We apply the Lasalle's invariance principle (see [7] and [10]) to the $\omega$-limit set

$$
\begin{array}{r}
\omega\left(y_{0}, y_{1}\right)=\left\{\left(z_{0}, z_{1}\right) \in \mathcal{H}:\left(z_{0}, z_{1}\right)=\lim _{n \rightarrow+\infty} \mathcal{S}\left(t_{n}\right)\left(y_{0}, y_{1}\right)\right. \\
\text { where } \left.t_{n} \rightarrow+\infty \text { as } n \rightarrow+\infty\right\}
\end{array}
$$

of the trajectory $\mathrm{O}^{+}\left(y_{0}, y_{1}\right)$. Note that

$$
\mathcal{S}(t)\left(y_{0}, y_{1}\right) \rightarrow \omega\left(y_{0}, y_{1}\right) \text { as } t \rightarrow+\infty \text {. }
$$

In order to show the asymptotic stability, it is sufficient to prove that the $\omega$-limit set reduces to $\{(0,0)\}$. For this, first (2.9) implies

$$
E(t)-E(s)+\int_{s}^{t}\left[k_{v, 0} y_{t}(0, \sigma) f\left(y_{t}(0, \sigma)\right)+k_{v, 1} y_{t}(1, \sigma) g\left(y_{t}(1, \sigma)\right)\right] d \sigma=0 .
$$

Second, let $\left(z_{0}, z_{1}\right) \in \omega\left(y_{0}, y_{1}\right) \subset D(A)$ and let $\left(z(t), z_{t}(t)\right)$ be the trajectory associated with $\left(z_{0}, z_{1}\right)$, so according to $(2.9)$ we obtain:

$$
\int_{s}^{t}\left[k_{v, 0} z_{t}(0, \sigma) f\left(z_{t}(0, \sigma)\right)+k_{v, 1} z_{t}(1, \sigma) g\left(z_{t}(1, \sigma)\right)\right] d \sigma=0
$$

therefore, we deduce from (1.8) that

$$
\left\{\begin{array} { l } 
{ k _ { v , 0 } z _ { t } ( 0 , t ) f ( z _ { t } ( 0 , t ) ) = 0 , } \\
{ k _ { v , 1 } z _ { t } ( 1 , t ) g ( z _ { t } ( 1 , t ) ) = 0 }
\end{array} \Rightarrow \left\{\begin{array}{rl}
z(0, t) & =c=\text { const } \\
k_{v, 1} z_{t}(1, t) & =0
\end{array} \quad \forall t \geq 0 .\right.\right.
$$


Thus $\omega\left(y_{0}, y_{1}\right)$ is included in the set of all initial data whose associated solution has constant energy, so $z$ verifies the following system:

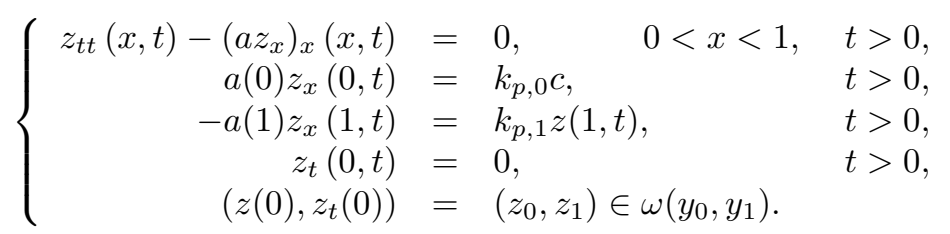

First, we prove that $\mathrm{c}=0$. For this, we consider the function $h(x)=1+$ $k_{p, 1} \int_{x}^{1} \frac{d s}{a(s)}$ and we multiply the first equation of (2.11) by $h$ and integrate in $x$ and $t$. A straightforward computation shows that

$$
\left[\int_{0}^{1} h(x) z_{t}(x, t) d x\right]_{0}^{T}=-\left[h(0) k_{p, 0}+k_{p, 1}\right] c T .
$$

The left term of (2.12) is bounded uniformly with respect to $T$. So, we divide (2.12) by $T$ and let it goes to infinity, we get $c=0$, then (2.11) yields

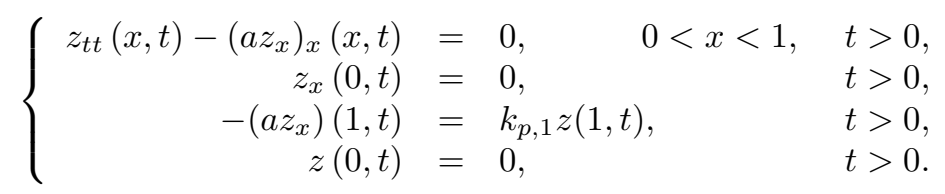

To achieve the proof, we distinguish two cases.

CASE $k_{p, 1}=0$. (2.13) can be written as follows

$$
\left\{\begin{aligned}
z_{t t}(x, t)-\left(a z_{x}\right)_{x}(x, t) & =0, & 0<x<1, & t>0 \\
z_{x}(0, t)=z_{x}(1, t) & =0, & & t>0 \\
z(0, t) & =0, & & t>0
\end{aligned}\right.
$$

This system has a unique solution $z=0$ (see [11]), so, the asymptotic stability follows for this case.

CASE $k_{p, 1}>0$. We multiply the first equation of (2.13) by $\varphi z_{x}$ and integrate in $x$ and $t$. Then one obtains

$$
\begin{gathered}
{\left[\int_{0}^{1} z_{t} \varphi z_{x} d x\right]_{0}^{T}-\frac{1}{2} \varphi(1) \int_{0}^{T} z_{t}^{2}(1, t) d t+\frac{1}{2} \int_{0}^{T} \int_{0}^{1} \varphi_{x} z_{t}^{2} d x d t} \\
=\frac{1}{2} \int_{0}^{T} \frac{\varphi(1)}{a(1)} k_{p, 1}^{2} z^{2}(1, t) d t-\frac{1}{2} \int_{0}^{T} \int_{0}^{1} a\left(\frac{\varphi}{a}\right)_{x} a z_{x}^{2} d x d t \\
{\left[\int_{0}^{1} z_{t} \varphi z_{x} d x\right]_{0}^{T}=\frac{1}{2} \int_{0}^{T} \frac{\varphi(1)}{a(1)} k_{p, 1}^{2} z^{2}(1, t) d t-\frac{1}{2} \int_{0}^{T} \int_{0}^{1} a \varphi_{x} \frac{1}{a} z_{t}^{2} d x d t} \\
-\frac{1}{2} \int_{0}^{T} \int_{0}^{1} a\left(\frac{\varphi}{a}\right)_{x} a z_{x}^{2} d x d t+\frac{1}{2} \varphi(1) \int_{0}^{T} z_{t}^{2}(1, t) d t
\end{gathered}
$$


The function $\varphi$ is chosen so that

$$
\varphi \in H^{1}(0,1), \quad-a(x) \varphi_{x} \geq k_{p, 1}, \quad \varphi(1) \geq 1 \text { and }-a\left(\frac{\varphi}{a}\right)_{x} \geq k_{p, 1} .
$$

With this choice and (1.2), we obtain

$$
\begin{gathered}
\frac{k_{p, 1}}{2 \underline{a}}\left[\int_{0}^{T}\left[\int_{0}^{1}\left(z_{t}^{2}+a(x) z_{x}^{2}\right) d x+k_{p, 1} z^{2}(1, t)\right] d t\right]+\frac{1}{2} \int_{0}^{T} z_{t}^{2}(1, t) d t \\
\leq\left[\int_{0}^{1} z_{t} \varphi z_{x} d x\right]_{0}^{T},
\end{gathered}
$$

which implies that

$$
\int_{0}^{T} E(t) d t \leq C(E(T)+E(0))=2 C E(0),
$$

where $C$ is a positive constant. The energy is constant, so

$$
E(T) \leq \frac{2}{T} C E(0) \quad \forall T>0,
$$

and then

$$
E(t)=0 \quad \forall t \geq 0,
$$

i.e. $z \equiv 0$ and so $z_{t} \equiv 0$. Thus $\omega\left(y_{0}, y_{1}\right)=\{(0,0)\}$. Therefore, the proof of Theorem 1.1. is complete.

2.2. Proof of Theorem 1.2. We adopt the method used by Conrad and Rao [4] but in our case we have two nonlinear feedback functions $f$ and $g$ satisfying more general growth conditions. We introduce the same functional defined in $[4]$

$$
\rho(t)=2 \int_{0}^{1} y_{t} \varphi y_{x} d x+C_{0} \int_{0}^{1} y_{t} \psi d x
$$

where $C_{0}$ is a positive constant and $\psi$ is the solution of the problem

$$
\left\{\begin{aligned}
\left(a \psi_{x}\right)_{x}(x, t) & =0, \quad 0<x<1 \\
\psi(0, t) & =y(0, t) \\
\psi(1, t) & =y(1, t)
\end{aligned}\right.
$$

We verify that the following inequalities hold

$$
\begin{aligned}
\int_{0}^{1} a \psi_{x} y_{x} d x & =\left[\int_{0}^{1} a^{-1}(s) d s\right]^{-1}[y(1, t)-y(0, t)]^{2} \geq 0, \\
\int_{0}^{1} \psi^{2} d x & \leq 5\left[y^{2}(0, t)+y^{2}(1, t)\right],
\end{aligned}
$$

similarly,

$$
\int_{0}^{1} \psi_{t}^{2} d x \leq 5\left[y_{t}^{2}(0, t)+y_{t}^{2}(1, t)\right]
$$


The function $\varphi$ is chosen so that

$$
\varphi \in H^{1}(0,1), \quad \varphi_{x} \geq 1, \quad a\left(\frac{\varphi}{a}\right)_{x} \geq 1, \quad \varphi(0)<0, \quad \varphi(1)>0
$$

and

$$
k_{p, 0} \varphi(0) a(1)+k_{p, 1} \varphi(1) a(0)=0 .
$$

We can show that there exist positive constants $K_{0}, K_{1}$ and $K_{2}$ such that for any $t \geq 0$,

$$
|\rho(t)| \leq K_{0} E(t)
$$

$$
\rho^{\prime}(t) \leq-E(t)+K_{1}\left[y_{t}^{2}(0, t)+y_{t}^{2}(1, t)\right]+K_{2}\left[f^{2}\left(y_{t}(0, t)\right)+g^{2}\left(y_{t}(1, t)\right)\right] .
$$

Given $\varepsilon>0$, we introduce (see [4]) the perturbed energy by

$$
E_{\varepsilon}(t)=E(t)+\varepsilon \rho(t)[E(t)]^{\frac{(p \vee q)-1}{2}} .
$$

In the formula (2.16), the exponent $p \vee q$ seems to be new. This together with the non-increasing of the energy $E(t)$ implies that for any $M>1$

$$
M^{-1 / 2}\left[E_{\varepsilon}(t)\right]^{\frac{(p \vee q)+1}{2}} \leq[E(t)]^{\frac{(p \vee q)+1}{2}} \leq M^{1 / 2}\left[E_{\varepsilon}(t)\right]^{\frac{(p \vee q)+1}{2}}
$$

with

$$
\varepsilon \leq K_{0}^{-1}[E(0)]^{\frac{1-(p \vee q)}{2}}\left(1-M^{-\frac{1}{(p \vee q)+1}}\right) .
$$

Now, we calculate the derivative of the perturbed energy $E_{\varepsilon}(t)$.

$$
E_{\varepsilon}^{\prime}(t)=E^{\prime}(t)+\varepsilon \frac{(p \vee q)-1}{2} \rho(t) E^{\prime}(t)[E(t)]^{\frac{(p \vee q)-3}{2}}+\varepsilon \rho^{\prime}(t)[E(t)]^{\frac{(p \vee q)-1}{2}}
$$

on the other hand, from (1.13), (1.14) and (2.15), one obtains

$$
\rho^{\prime}(t) \leq-E(t)+K_{3} y_{t}^{2}(0, t)+K_{4} y_{t}^{2}(1, t)
$$

where

$$
K_{3}=K_{1}+K_{2} C_{2}^{2} \quad \text { and } \quad K_{4}=K_{1}+K_{2} C_{4}^{2} .
$$

Plugging (1.7), (2.14) and (2.19) into equation (2.18), one obtains

$$
\begin{aligned}
E_{\varepsilon}^{\prime}(t) \leq & {\left[-1+\varepsilon \frac{(p \vee q)-1}{2} K_{0}[E(0)]^{\frac{(p \vee q)-1}{2}}\right] } \\
& {\left[k_{v, 0} y_{t}(0, t) f\left(y_{t}(0, t)\right)+k_{v, 1} y_{t}(1, t) g\left(y_{t}(1, t)\right)\right] } \\
& +\varepsilon[E(t)]^{\frac{(p \vee q)-1}{2}}\left[K_{3} y_{t}^{2}(0, t)+K_{4} y_{t}^{2}(1, t)\right]-\varepsilon[E(t)]^{\frac{(p \vee q)+1}{2}} .
\end{aligned}
$$

Now we distinguish the case $p \vee q=1$ and $p \vee q>1$.

(i) Case $p \vee q=1$. In this case (2.20) yields

$$
\begin{aligned}
E_{\varepsilon}^{\prime}(t) \leq & \left(\varepsilon \frac{K_{4}}{C_{3}}-k_{v, 1}\right) y_{t}(1, t) g\left(y_{t}(1, t)\right) \\
& +\left(\varepsilon \frac{K_{3}}{C_{1}}-k_{v, 0}\right) y_{t}(0, t) f\left(y_{t}(0, t)\right)-\varepsilon E(t) .
\end{aligned}
$$


If we choose $\varepsilon \leq \varepsilon_{0}=\min \left(\frac{C_{3} k_{v, 1}}{K_{4}}, \frac{C_{1} k_{v, 0}}{K_{3}}\right)$, then from (1.8) and (2.17), the previous inequality becomes

$$
E_{\varepsilon}^{\prime}(t) \leq-\varepsilon E(t) \leq-\varepsilon M^{-1 / 2} E_{\varepsilon}(t)
$$

Now, integrating $\frac{E_{\varepsilon}^{\prime}(t)}{E_{\varepsilon}(t)}$ on $[0, t]$, and using $(2.17)$, we get

$$
E(t) \leq M E(0) e^{-\omega t}, \quad \forall t \geq 0,
$$

where $\omega=\varepsilon M^{-1 / 2}$, with $\varepsilon \leq \min \left(\varepsilon_{0}, K_{0}^{-1}\left(1-M^{-\frac{1}{2}}\right)\right)$.

(ii) Case $p \vee q>1$.

If $y_{t}^{2}(1, t)>1$, it follows from hypothesis (1.8) and (1.14) that

$$
\varepsilon K_{4}[E(t)]^{\frac{(p \vee q)-1}{2}} y_{t}^{2}(1, t) \leq \frac{\varepsilon K_{4}}{C_{3}}[E(0)]^{\frac{(p \vee q)-1}{2}} y_{t}(1, t) g\left(y_{t}(1, t)\right) .
$$

However, while $y_{t}^{2}(1, t) \leq 1$, by Young's inequality, we have for any parameter $\delta>0$

$$
\begin{aligned}
\varepsilon K_{4}[E(t)]^{\frac{(p \vee q)-1}{2}} y_{t}^{2}(1, t) \leq & \frac{2 \varepsilon}{(p \vee q)+1}\left(K_{4} \delta\right)^{\frac{(p \vee q)+1}{2}}\left|y_{t}(1, t)\right|^{(p \vee q)+1} \\
& +\varepsilon \frac{(p \vee q)-1}{(p \vee q)+1} \delta^{-\frac{(p \vee q)+1}{(p \vee q)-1}}[E(t)]^{\frac{(p \vee q)+1}{2}} .
\end{aligned}
$$

Since $\min \left(\left|y_{t}(1, t)\right|,\left|y_{t}(1, t)\right|^{q}\right)=\left|y_{t}(1, t)\right|^{q}$, and $p \vee q \geq q$, we have

$$
\left|y_{t}(1, t)\right|^{1+(p \vee q)} \leq\left|y_{t}(1, t)\right|^{q} \leq \frac{1}{C_{3}} y_{t}(1, t) g\left(y_{t}(1, t)\right) .
$$

This implies that

$$
\begin{aligned}
\varepsilon K_{4}[E(t)]^{\frac{(p \vee q)-1}{2}} y_{t}^{2}(1, t) \leq & \frac{2 \varepsilon}{p \vee q+1} \frac{\left(K_{4} \delta\right)^{\frac{(p \vee q)+1}{2}}}{C_{3}} y_{t}(1, t) g\left(y_{t}(1, t)\right) \\
& +\varepsilon \frac{(p \vee q)-1}{(p \vee q)+1} \delta^{-\frac{(p \vee q)+1}{(p \vee q)-1}}[E(t)]^{\frac{(p \vee q)+1}{2}} .
\end{aligned}
$$

Combining (2.21) and (2.22), one has

$$
\begin{aligned}
\varepsilon K_{4}[E(t)]^{\frac{(p \vee q)-1}{2}} y_{t}^{2}(1, t) \leq & \varepsilon \frac{(p \vee q)-1}{(p \vee q)+1} \delta^{-\frac{(p \vee q)+1}{(p \vee q)-1}}[E(t)]^{\frac{(p \vee q)+1}{2}} \\
& +\varepsilon K_{5} y_{t}(1, t) g\left(y_{t}(1, t)\right)
\end{aligned}
$$

where $K_{5}=\frac{K_{4}}{C_{3}}[E(0)]^{\frac{(p \vee q)-1}{2}}+\frac{2}{(p \vee q)+1} \frac{\left(K_{4} \delta\right)^{\frac{(p \vee q)+1}{2}}}{C_{3}}$. Similarly, we can show that

$$
\begin{aligned}
\varepsilon K_{3}[E(t)]^{\frac{(p \vee q)-1}{2}} y_{t}^{2}(0, t) \leq & \varepsilon \frac{(p \vee q)-1}{(p \vee q)+1} \delta^{-\frac{(p \vee q)+1}{(p \vee q)-1}}[E(t)]^{\frac{(p \vee q)+1}{2}} \\
& +\varepsilon K_{6} y_{t}(0, t) f\left(y_{t}(0, t)\right),
\end{aligned}
$$


with $K_{6}=\frac{K_{3}}{C_{1}}[E(0)]^{\frac{(p \vee q)-1}{2}}+\frac{2}{(p \vee q)+1} \frac{\left(K_{3} \delta\right) \frac{(p \vee q)+1}{2}}{C_{1}}$. Inserting (2.23) and (2.24) into (2.20), we obtain

$$
\begin{aligned}
E_{\varepsilon}^{\prime}(t) \leq & \varepsilon\left[2 \frac{(p \vee q)-1}{(p \vee q)+1} \delta^{-\frac{(p \vee q)+1}{(p \vee q)-1}}-1\right][E(t)]^{\frac{(p \vee q)+1}{2}} \\
& +\left[-k_{v, 0}+\varepsilon k_{v, 0} \frac{(p \vee q)-1}{2} K_{0}[E(0)]^{\frac{(p \vee q)-1}{2}}+\varepsilon K_{6}\right] y_{t}(0, t) f\left(y_{t}(0, t)\right) \\
& +\left[-k_{v, 1}+\varepsilon k_{v, 1} \frac{(p \vee q)-1}{2} K_{0}[E(0)]^{\frac{(p \vee q)-1}{2}}+\varepsilon K_{5}\right] y_{t}(1, t) g\left(y_{t}(1, t)\right) .
\end{aligned}
$$

This implies that

$$
E_{\varepsilon}^{\prime}(t) \leq-\mu \varepsilon[E(t)]^{\frac{(p \vee q)+1}{2}}
$$

provided that $\delta$ is chosen such that for some $\mu>0,2 \frac{(p \vee q)-1}{(p \vee q)+1} \delta^{-\frac{(p \vee q)+1}{(p \vee q)-1}}-1 \leq$ $-\mu<0$, and $\varepsilon$ is chosen as follows

$$
-k_{v, i}+\varepsilon\left[K_{6-i}+k_{v, i} \frac{(p \vee q)-1}{2} K_{0}[E(0)]^{\frac{(p \vee q)-1}{2}}\right] \leq 0,
$$

where $i=0,1$. Combining (2.17) and (2.25), we get

$$
E_{\varepsilon}^{\prime}(t) \leq-\mu \varepsilon M^{-1 / 2}\left[E_{\varepsilon}(t)\right]^{\frac{(p \vee q)+1}{2}} .
$$

Finally, solving the differential inequality (2.26) and using (2.17), we obtain

$$
\begin{array}{r}
E(t) \leq M E(0)(1+\omega t)^{-\frac{2}{(p \vee q)-1}} \\
\text { with } \omega=\frac{(p \vee q)-1}{2} \mu \varepsilon M^{-\frac{p \vee q}{(p \vee q)+1}}[E(0)]^{\frac{(p \vee q)-1}{2}} .
\end{array}
$$

This completes the proof of Theorem 1.2.

REMARK 2.2. The result of Theorem 1.2 (ii) remains true if we replace (1.14) with

$$
\begin{aligned}
& C_{1}|x| \leq|f(x)| \leq C_{2} \max \left(|x|,|x|^{\frac{1}{p}}\right) \\
& C_{3}|x| \leq|g(x)| \leq C_{4} \max \left(|x|,|x|^{\frac{1}{q}}\right)
\end{aligned}
$$

or with

$$
\begin{aligned}
C_{1}|x| & \leq|f(x)| \leq C_{2} \max \left(|x|,|x|^{\frac{1}{p}}\right), \\
C_{3} \min \left(|x|,|x|^{q}\right) & \leq|g(x)| \leq C_{4}|x|
\end{aligned}
$$

where $(p, q) \in\left[1,+\infty\left[^{2}\right.\right.$ with $\max (p, q)=p \vee q>1$.

For the cases (2.27) and (2.28) the energy decay estimates is: given any $M>1$, there exists a constant $\omega>0$ depending on $E(0)$ such that

$$
E(t) \leq M E(0)(1+\omega t)^{-\frac{2}{(p \vee q)-1}}, \quad \forall t \geq 0 .
$$




\section{REFERENCES}

[1] B. Chentouf, C. Z. Xu and G. Sallet, On the stabilization of a vibrating equation, Nonlinear Anal. 39 (2000), 537-558.

[2] G. Chen and H. K. Wang, Asymptotic behavior of solutions of the one-dimensional wave equation with a nonlinear boundary stabilizer, SIAM J. Control Optim. 27 (1989), 758-775.

[3] M. Cherkaoui, Sur quelques problèmes de stabilisation de l'équation des ondes avec contrôle frontière, Thèse d'État, Université Abdelmalek ESSAADI, Faculté des Sciences, Tetouan, Morocco, 2002.

[4] F. Conrad and B. Rao, Decay of solutions of the wave equation in a star-shaped domain with nonlinear boundary feedback, Asymptotic Anal. 7 (1993), 159-177.

[5] C. M. Dafermos and M. Slemrod, Asymptotic behaviour of nonlinear contraction semigroups, J. Functional Analysis 13 (1973), 97-106.

[6] A. Haraux, Semi-linear hyperbolic problems in bounded domains, Math. Rep. 3 (1987), i-xxiv and 1-281.

[7] A. LaSalle and S. Lefschetz, Stability by Lyapounov's direct method, with applications, Academic Press, New York-London, 1961.

[8] I. Lasiecka, Stabilization of wave equations with nonlinear dissipative damping on the boundary, in: Proc. $26^{\text {th }}$ IEEE Conf. on decision and Control, Los Angeles, CA, 1987, 2348-2349.

[9] I. Lasiecka, Stabilization of a wave and plate - like equations with nonlinear dissipative damping on the boundary, Applied Mathematics Report RM-88-05, University of Virginia, Charlottesville, VA, March 1988.

[10] M. Slemrod, Feedback stabilization of a linear control system in Hilbert space with an priori bounded control, Math. Control Signals Systems 2 (1989), 265-285.

[11] E. Zuazua, Some remarks on the boundary stabilizability of the wave equation, in: Control of Boundaries and Stabilization, ed. J. Simon, Lecture Notes in Control and Inform. Sci. 125, Springer-Verlag, Berlin, 1989.

\section{Cherkaoui}

Université Moulay Ismail

Département de Mathematiques

Faculté des Sciences et Techniques

B. P. 509, Errachidia

Maroc

E-mail: mad_cherkaoui@yahoo.fr

N. Yebari

Université Abdelmalek Essaadi

Département de Mathematiques

Faculté des Sciences

B. P. 2121, Tetouan

Maroc

E-mail: nyebari@hotmail.com

Received: 11.5.2007.

Revised: 3.3.2008. 